\title{
AEG-1 deletion promotes cartilage repair and modulates bone remodeling-related cytokines via TLR4/MyD88/NF- $\kappa B$ inhibition in ovariectomized rats with osteoporosis
}

\author{
Yuan Zhang", Qing Zhao" \\ Department of Rheumatology, Henan University Huai He Hospital, Kaifeng City, China \\ Contributions: (I) Conception and design: Y Zhang; (II) Administrative support: Q Zhao; (III) Provision of study materials or patients: All authors; \\ (IV) Collection and assembly of data: All authors; (V) Data analysis and interpretation: All authors; (VI) Manuscript writing: All authors; (VII) Final \\ approval of manuscript: All authors. \\ \#These authors contributed equally to this work. \\ Correspondence to: Yuan Zhang. Henan University Huai He Hospital, No. 185 Ximen Street, Gulou District, Kaifeng City, China. \\ Email: osfnn811456@sina.cn.
}

Background: Osteoporosis is a systemic skeletal disorder that can impact a variety of bones throughout the
body. Astrocyte-elevated gene-1 (AEG-1) is involved in multiple pro-tumorigenic functions and participates
in various inflammatory reactions. However, whether it has an impact on osteoporosis-related cartilage
repair and bone remodeling remains unknown.
Methods: We utilized an ovariectomy mouse model with AEG-1 deletion to investigate the role of AEG-
1 in osteoporosis. The mRNA level of AEG-1 was detected by RT-PCR, bone markers, bone volume/total
volume (BV/TV), trabecular bone surface/bone volume (BSA/BV) and trabecular bone thickness (Tb. Th) were
detected by micro computed tomography ( $\mu \mathrm{CT}$ ), bone injury was observed by HE and alcian blue staining.
The contents of IL-6, IL-17, iNOS and IL-10 in peripheral blood of the three groups were detected by ELISA.
The expression of OSX, coila1, OC, TLR4, MyD88 and NF- $\mathrm{kB}$ were detected by Western Blot.
Results: $\mu \mathrm{CT}$ revealed increased bone volume in the AEG-1 knockout (KO) ovariectomy (OVX) group
compared to the wildtype (WT) OVX group 4 weeks after surgery, indicating restored bone formation
after AEG-1 deletion. Flow sorting revealed that AEG-1 deletion inhibited the production of inflammatory
factors. Western blot demonstrated activation of the TLR4/MyD88/NF- $\mathrm{BB}$ pathway after LPS exposure,
which was reduced by AEG-1 deletion. AEG-1 deletion also improved lipopolysaccharide (LPS) induced
adverse reactions.

Conclusions: Taken together, these findings indicate that AEG-1 deletion improves cartilage repair and bone remodeling during osteoporosis, which may partly occur through the inhibition of the TLR4/MyD88/ $\mathrm{NF}-\kappa \mathrm{B}$ signaling pathway.

Keywords: Osteoporosis; IL-10; lipopolysaccharide (LPS); bone loss; osteoclastogenesis; micro-CT

Submitted Jul 16, 2020. Accepted for publication Oct 11, 2020.

doi: $10.21037 / \mathrm{atm}-20-5842$

View this article at: http://dx.doi.org/10.21037/atm-20-5842

\section{Introduction}

Osteoporosis is a systemic skeletal disorder that can impact a variety of bones throughout the body, and is primary a health problem among the elderly. Osteoporosis is characterized by low bone mineral density (BMD), abnormal microstructure of bone tissue, increased bone fragility, and high risk of fracture (1). It is well known that the main form of bone metabolism is bone remodeling. Bone remodeling is mainly performed by osteoblasts and osteoclasts. Osteoclasts absorb old bone and promote the 
degradation of old bone, whilst osteoblasts synthesize new bone and maintain the source of new bone. The delicate balance between bone resorption and bone formation is essential for maintaining healthy bone mass and mitigating the risk of osteoporosis $(2,3)$. Osteoporosis is prevalent among women, predominantly in postmenopausal women. Currently, drugs used for postmenopausal osteoporosis include bisphosphonates, selective estrogen receptor modulators, calcitonin, estrogen and recombinant human parathyroid hormone fragments. Adverse reactions, such as typical femoral fractures, bisphosphonate-related osteonecrosis of the jaw, hypercalcemia, thromboembolism, vaginal bleeding, and even breast cancer and endometrial cancer, significantly limit the widespread use of these drugs. Hence, novel treatment strategies are required.

Toll-like receptors (TLRs) belong to pattern recognition receptors (PRRs) and are a critical part of the innate immune system (4). Toll-like receptor 4 (TLR4) was the first discovered member of the TLR family. Previous studies revealed that TLR4 mediates the inflammatory response in the myocardium, and inflammatory signaling pathways mediated by TLR4 play a major role in myocardial infarction, myocarditis and ischemia-reperfusion injury (5-7). Several studies have revealed that TLR4/ MyD88/NF- $\mathrm{KB}$ signaling induces the production of proinflammatory factors, causing further myocardial tissue injury $(8,9)$. Wang et al. reported that TLR4/MyD88/ $\mathrm{NF}-\kappa \mathrm{B}$ signaling could exacerbate myocardial damage by accelerating the inflammatory cascade through activation of the nod-like receptor protein 3 (NLRP3) (10). Vijayan

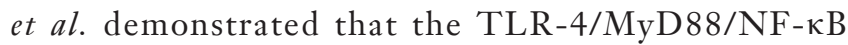
signaling pathway contributes to osteoclast development, and methionine treatment could mitigate postmenopausal osteoporosis by downregulating the TLR-4/MyD88/NF$\kappa \mathrm{B}$ signalling pathway (11).

Astrocyte-elevated gene-1 (AEG-1), also known as Metadherin or Lyric, was originally identified as a late response gene induced by human immunodeficiency virus (HIV)-1 (12,13). It is involved in multiple pro-tumorigenic functions, including malignant transformation, angiogenesis and metastasis. Recently, studies have shown that AEG-1 is markedly overexpressed in multiple types of human cancers, including hepatocellular carcinoma, gastrointestinal cancer, breast cancer and malignant glioma (14-16). AEG-1 has also been linked to pro-tumorigenic signaling transduction pathways, including the AKT, NF- $\kappa \mathrm{B}, \mathrm{PI} 3 \mathrm{~K}-\mathrm{AKT}$ and Wnt pathways $(17,18)$. Wang et al. reported that Cs-gPLLD-FA nanoparticles are a promising system to treat osteosarcoma through the effective delivery of AEG1 siRNA (19). However, its functional significance and underlying molecular mechanisms in osteoporosis remain to be elucidated.

The pathogenesis of osteoporosis is multifactorial and the underlying molecular and cellular mechanisms remain to be understood. In our study, wildtype (WT) Sprague Dawley (SD) rats and AEG-1 knockout $(\mathrm{KO})$ SD rats were used to establish an ovariectomy model. In osteoporosis caused by estrogen deficiency, trabecular bone morphometry was aberrant, the expression of the bone specific transcription factors Osx, CoI1a1 and OC decreased, levels of inflammatory factors IL-6, IL-17, iNOS increased and the TLR-4/MyD88/NF- $\kappa B$ signaling pathway was activated. It is well known that lipopolysaccharide (LPS) can induce bone loss by enhancing the bone resorption or inhibiting bone formation. Of note, AEG-1 deletion mitigated these aberrant phenomena and reversed LPS induced damage. Therefore, this study suggests that AEG-1 may be a novel therapeutic target for osteoporosis. We present the following article in accordance with the ARRIVE reporting checklist (available at http://dx.doi.org/10.21037/atm-205842).

\section{Methods}

\section{Animals}

WT SD rats and AEG-1 KO SD rats (mature females, six weeks old) were purchased from Cyagen Biosciences Co., Ltd. (Suzhou, Jiangsu, China). All rats were kept in polypropylene cages with a 12 -h light/dark cycle under $21 \pm 4^{\circ} \mathrm{C}$ and allowed free access to food and water.

All animal experiment protocols were approved by Roslin Institute's Animal Users Committee (A650). All animal experiments were performed in accordance with the International Guiding Principles for Biomedical Research Involving Animals issued by the Council for the International Organizations of Medical Sciences.

\section{Osteoporosis model}

At 10 weeks of age, rats were divided into four groups. The WT sham group (n=4), WT-OVX group (n=4), AEG-1 KO group $(n=4)$ and AEG-1 KO OVX group $(n=4)$ underwent bilateral ovariectomy. The surgery was performed as previously described (11). After 4 weeks of regular feeding, tissues were collected following exsanguination under 
anesthesia.

To assess the role of AEG-1 in the inflammatory component of osteoporosis, lipopolysaccharide (LPS) induced experiments were performed by modifying the protocol previously described (20). To establish an inflammatory bone loss model, the WT OVX group $(n=4)$ and the AEG-1 KO OVX group $(n=4)$ were injected intraperitoneally with LPS (20 $\mu \mathrm{g}$ LPS in $10 \mu \mathrm{L}$ of PBS) after one week of regular feeding (WT OVX + LPS group and AEG-1 KO OVX + LPS group). The injections were repeated 3 times each week for 3 weeks. Three days after last injection time, tissues were collected following exsanguination under anesthesia. For each group, body weights were assessed before sacrifice. Femurs and tibiae were immediately fixed in 4\% (w/v) paraformaldehyde for micro-computed tomography (CT) and bone histomorphometry analyses.

\section{RT-PCR}

Total RNA of organs and brain tissue were extracted using a TRIzolTM kit (Invitrogen, Waltham, MA, USA) following the manufacturer's instructions. After quantification, $0.5 \mu \mathrm{g}$ RNA from each sample was used to synthesize cDNA with a cDNA Synthesis kit (Invitrogen, Waltham, MA, USA). AEG-1 mRNA expression levels were determined using the SYBRPCR MasterMix kit (TaKaRa, Shiga, Japan) with GAPDH as an internal reference to normalize individual gene expression according to the $2^{-\Delta \Delta \mathrm{Ct}}$ method.

\section{Micro-CT analysis}

Rat femurs were dissected without soft tissue, fixed in $4 \%$ paraformaldehyde for 24 hours, and scanned with microCT. Parameters set: voltage was $60 \mathrm{kV}$, current was $220 \mu \mathrm{A}$, exposure time was $1,500 \mathrm{~ms}$, and effective pixel size was $8.89 \mu \mathrm{m}$ (21). The high-resolution Inveon microtomography (Siemens, Munich, Germany) was used to evaluate the morphometry of cartilage and trabecular bones. In order to implement further quantitative analysis, bone volume/ total volume (BV/TV), bone surface area/BV (BSA/BV), trabecular thickness (Tb. Th) and trabecular bone number (Tb. N) were analyzed using Inveon Research Workplace.

\section{Histological analysis}

Right femurs were stored in 10\% formaldehyde fixative for 24 hours at room temperature for histomorphometric analysis. Samples were placed in $10 \%$ formic acid for 28 days to decalcify, then routinely examined using an optical microscope. The decalcified fixed right femur was cross-sectioned into $3 \mu \mathrm{m}$ thick sections using a microtome. Hematoxylin and eosin ( $\mathrm{H} \& \mathrm{E})$ staining and Alcian blue staining were used to observe femoral injury under light microscopy, and 5 random fields per slice were imaged (22).

\section{ELISA}

Peripheral blood samples of rats were extracted and stored at $-80{ }^{\circ} \mathrm{C}$ until use. Levels of IL-6, IL-17, iNOS and IL-10 in peripheral blood were measured using standard sandwich ELISA kits (R\&D Systems) according to the manufacturer's instructions.

\section{Cell sorting}

The numbers of $\mathrm{CD}^{+} \mathrm{IL}-17^{+}$and $\mathrm{CD}^{+} \mathrm{IL}-10^{+}$positive cells in peripheral blood were detected by flow sorting. Cells were sorted on a FACSAria cell sorter (BD Biosciences). Purity of the sorted populations was greater than $98 \%$.

\section{Western blot analysis}

Protein from femurs was isolated with RIPA buffer and was quantified using a bicinchoninic acid kit (Qiaoyu Biotechnology Co., Ltd., Shanghai, China). Total protein samples $(20 \mu \mathrm{g})$ were loaded onto $10 \%$ sodium dodecyl sulfate polyacrylamide gel electrophoresis (SDS PAGE) loading buffer and were subsequently transferred to polyvinylidene difluoride (PVDF) membranes.

The membrane was blocked with $5 \%$ skim milk for 1 hour at room temperature, followed by incubation with the primary antibodies and GAPDH overnight at $4{ }^{\circ} \mathrm{C}$. Primary antibodies are as follows: Osx (ab209484, 1:1,000), CoI1A1 (ab64883, 1:1,000), OC (ab216327, 1:1,000), TLR4(ab13867, 1:500), p-TLR4(ab13556, 1:500), MyD88 (ab219413, 1:1,000), p-MyD88 (ab2064, 1:500), NF-кB p65 (ab16502, 1:500), p-NF-кB p65 (ab222494, 1:500). After washing twice with TBST, the membranes were incubated with secondary antibodies (ZSGB BIO, ZB2305, ZB2301) for 1 hour at room temperature. Protein bands were detected using an ECL detection kit (Thermo Scientific, 34077) and imaged.

\section{Statistical analysis}

Data are presented as mean \pm SD. Multiple comparisons of parametric data were measured by one-way ANOVA, followed by Bonferroni's multiple comparisons test between group pairs (SPSS 21.0, SPSS, Inc.). $\mathrm{P}<0.05$ was considered statistically significant. 

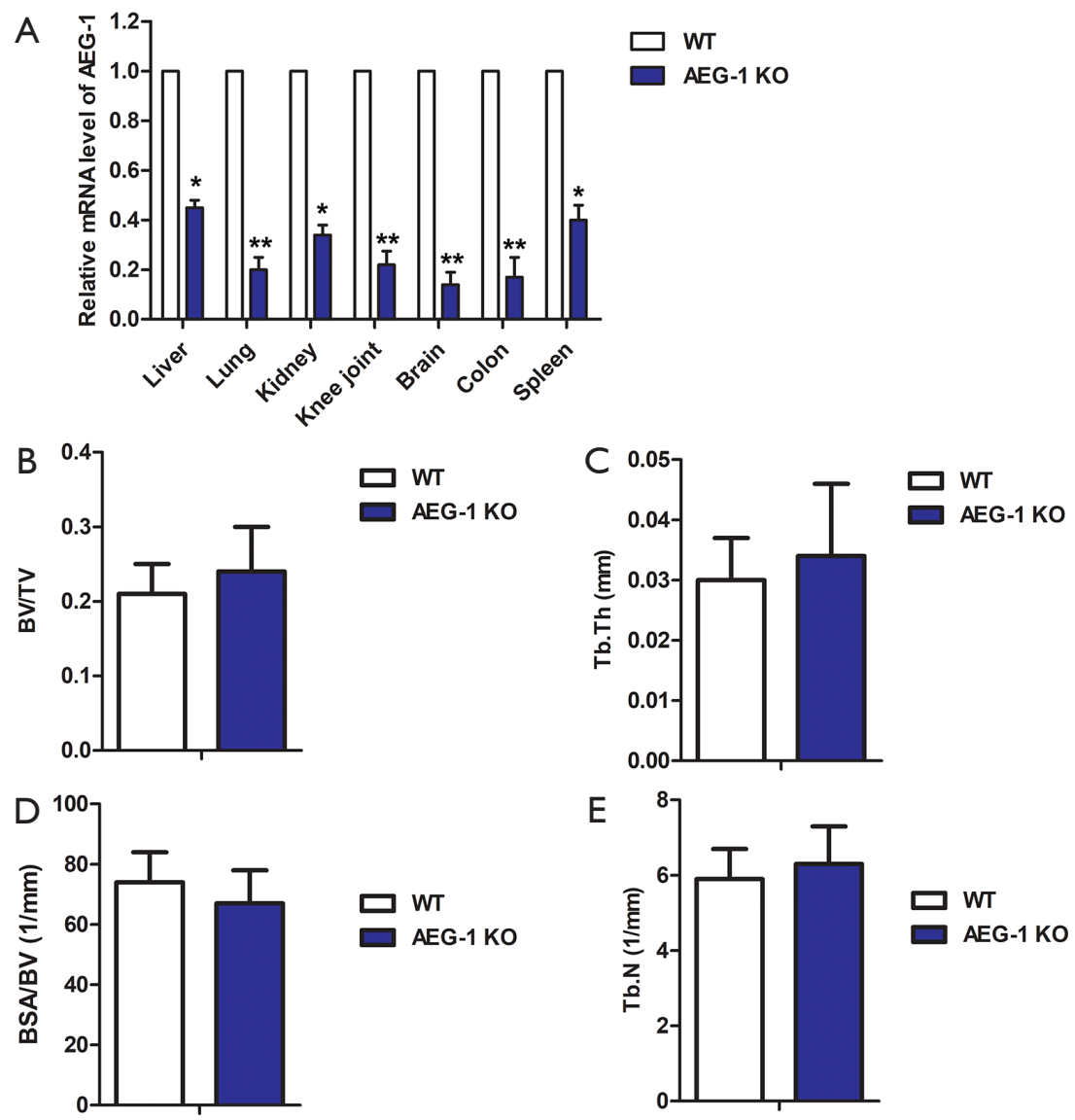

Figure 1 Establishment of AEG-1 deletion in rats. (A) Relative mRNA expression levels of AEG-1 in WT and AEG-1 KO tissues. Trabecular markers including BV/TV (B), Tb. Th (C), BSA/BV (D) and Tb. N (E) between WT and AEG-1 KO groups have no significant difference. The results are presented as mean $\pm \mathrm{SD}$ and represent the average of three individual experiments. * $\mathrm{P}<0.05 ;{ }^{* *}, \mathrm{P}<0.01$ versus the WT sham group $(n=4)$.

\section{Results}

\section{Establishment of AEG-1 KO rats}

To verify the successful generation of AEG-1 KO, relative mRNA expression levels of AEG-1 and trabecular bone morphometry in the WT group and AEG-1 KO group were measured. As shown in Figure 1A, compared with the WT group, relative mRNA expression levels of AEG-1 in organs, knee joints and brain were significantly decreased in the AEG-1 KO group $\left({ }^{*} \mathrm{P}<0.05,{ }^{*} \mathrm{P}<0.01\right)$. As depicted in Figure 1B,C,D,E, micro-CT showed normal trabecular bone morphometry in the WT group and the AEG-1 KO group. These results established that the AEG-1 KO was successful.

\section{AEG-1 deletion ameliorates OVX-induced bone loss in vivo}

As shown in Figure 2A, relative mRNA expression levels of AEG-1 in the WT OVX group significantly increased compared with the WT sham group. However, compared with the WT OVX group, relative mRNA expression levels of AEG-1 in the AEG-1 KO OVX group were significantly decreased. These results indicated that AEG-1 mRNA levels were overexpressed in OVX rats.

BV/TV, Tb. Th, and Tb.N values were lower in the distal femurs of WT OVX rats, while the value of BSA/BV was higher (Figures 2B,C,D,E). Conversely, bone mass was elevated in the AEG-1 KO OVX group compared with the 

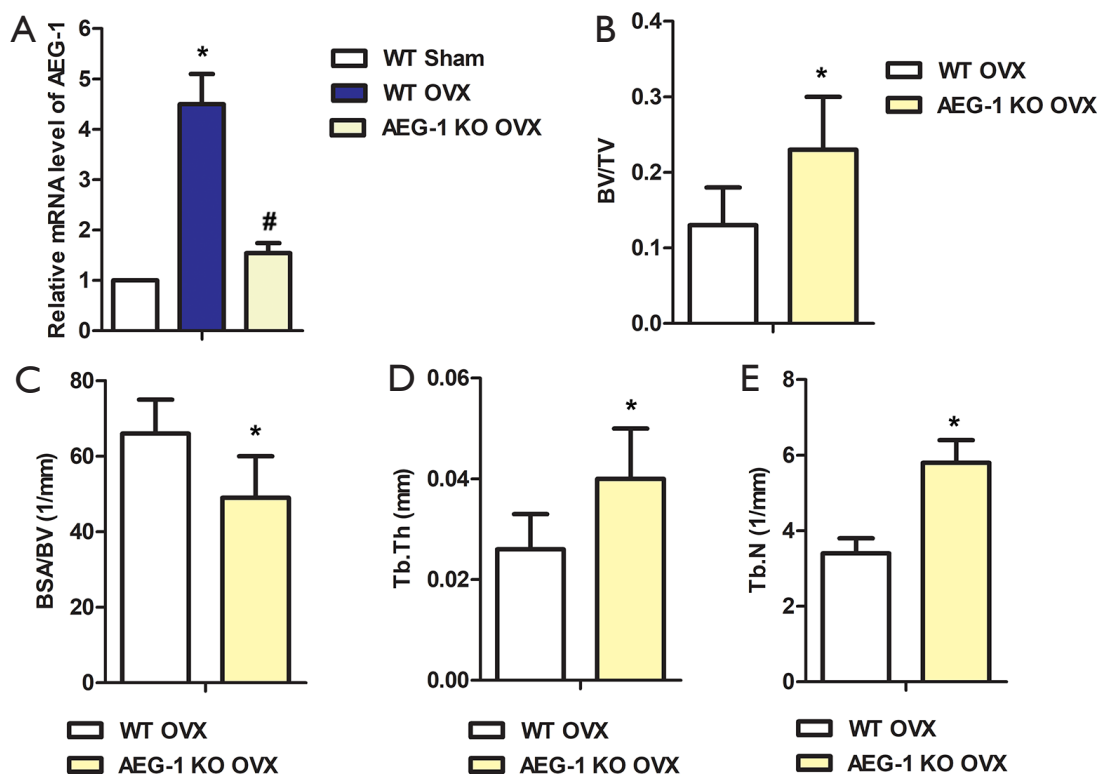

Figure 2 AEG-1 deletion ameliorates OVX-induced bone loss in vivo. (A) Relative mRNA expression levels of AEG-1 in WT sham, WT OVX and AEG-1 KO OVX tissues. Trabecular markers including (B) BV/TV (C) BSA/BV (1/mm) (D) Tb.Th (mm) (E) Tb.N (1/mm) were assessed. The results are presented as mean $\pm \mathrm{SD}$ and represent the average of three individual experiments. *, $\mathrm{P}<0.05$ versus the $\mathrm{WT}$ sham group ( $\mathrm{n}=4) ;{ }^{*}, \mathrm{P}<0.05$ versus the WT OVX group $(\mathrm{n}=4)$.

WT OVX group (Figure 2B,C,D,E). Taken together, these findings suggest that AEG-1 KO ameliorates OVX-induced bone loss in vivo.

\section{AEG-1 deletion promotes cartilage repair and modulates bone remodeling-related cytokines}

To further confirm the effect of AEG-1 deletion on osteoporosis, histological analysis was performed. As shown in Figure 3A, hydrated nucleus pulposus with smooth cartilage and surrounding extracellular matrix were observed in the WT sham group. However, in the WT OVX group, shrunken nucleus pulposus, cluster formation and cleft formation with fissures were observed. In the AEG-1 KO OVX group, this aberrant histological phenomena was reversed. These results indicated that OVX-associated changes in histological morphology were restored by deletion of AEG-1, showing a normal number of notochordal cells in the nucleus pulposus and minor mucoid degeneration. As shown in Figure $3 B$ and $C$, the percentage of trabecular bone and cartilage area were significantly decreased in the WT OVX group compared to the WT sham group. Notably, AEG-1 KO was able to reverse this. Furthermore, increased protein levels of Osx, CoI1A1 and OC were detected by western blotting (Figure 3D,E). Taken together, AEG1 deletion promotes cartilage repair and modulates bone remodeling-related cytokines.

\section{AEG-1 deletion ameliorates inflammatory damage in ovariectomized rats with osteoporosis}

To evaluate the effect of AEG-1 KO on the inflammatory component of osteoporosis, the levels of IL-6, IL-17, iNOS and IL-10 in the peripheral blood were detected using ELISA. As shown in Figure 4A,B,C, the concentrations of IL-6, IL-17 and iNOS in the WT OVX group were significantly greater than the WT sham group. However, compared with the WT OVX group, the concentrations of IL-6, IL-17 and iNOS in the AEG-1 KO OVX group were significantly lower. The concentration of IL-10 in the AEG-1 KO OVX group was significantly greater compared to the WT OVX group (Figure 4D).

To further confirm these experimental results, the number of $\mathrm{CD} 4^{+} \mathrm{IL}-17^{+}$and $\mathrm{CD} 4^{+} \mathrm{IL}-10^{+}$positive cells in peripheral blood were counted by flow sorting. Compared with the WT sham group, the percentage of $\mathrm{CD}^{+} \mathrm{IL}-$ $17^{+}$positive cells significantly increased in the WT OVX group, however, AEG-1 deletion mitigated this (Figure 4E). 
A
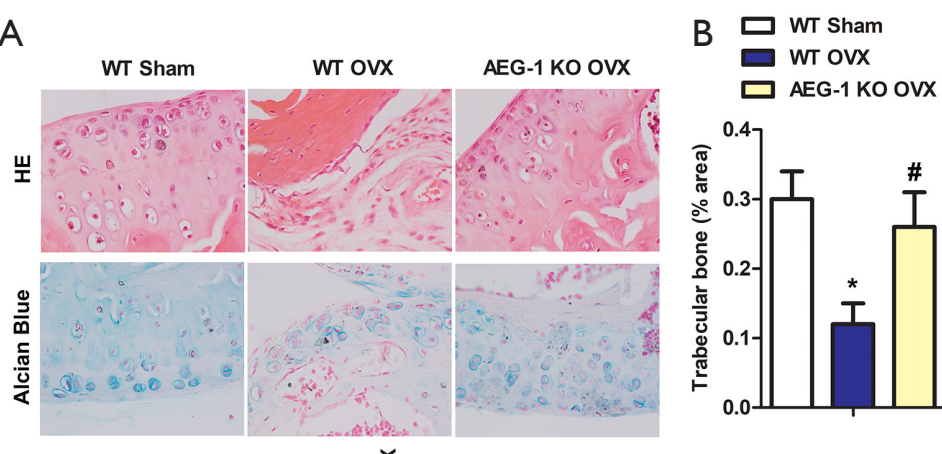

$E$
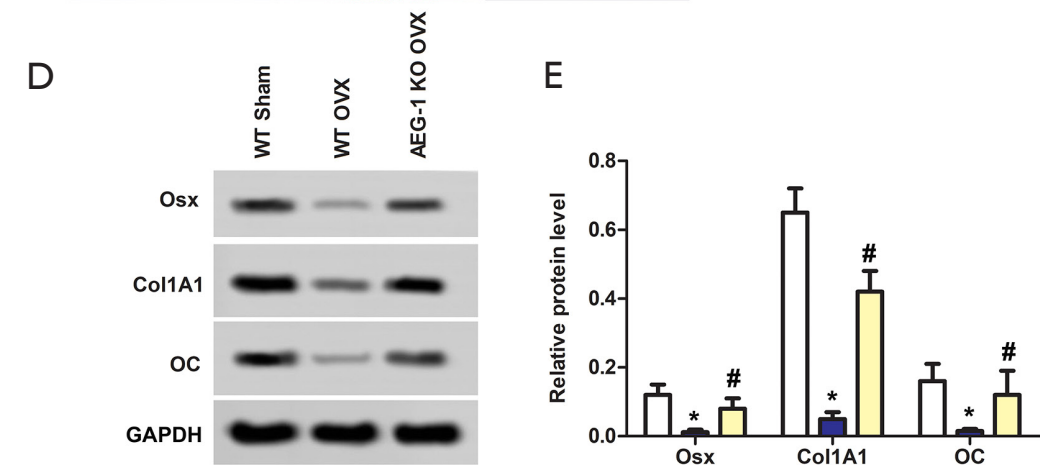
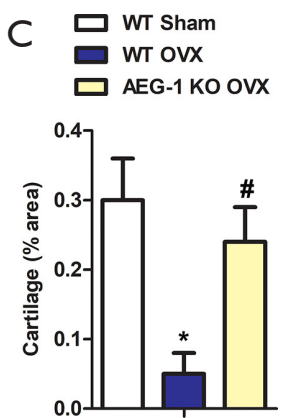

Figure 3 AEG-1 deletion promotes cartilage repair and modulates bone remodeling-related cytokines. (A) Representative images of H\&E staining (magnification $\times 200$ ) and Alcian Blue staining (magnification $\times 200$ ). (B) AEG-1 deletion enhances the area of trabecular bone. (C) AEG-1 deletion promotes cartilage repair. (D) Representative images of western blot bands; (E) AEG-1 deletion enhances relative protein levels of Osx, Col1a1 and OC. The results are presented as mean \pm SD and represent the average of three individual experiments. * $\mathrm{P}<0.05$ versus the WT sham group $(\mathrm{n}=4) ;{ }^{*}, \mathrm{P}<0.05$ versus the WT OVX group $(\mathrm{n}=4)$.

As shown in Figure $4 \mathrm{~F}$, the percentage of $\mathrm{CD}^{+} \mathrm{IL}-10^{+}$ positive cells was significantly increased in the WT sham group and the WT OVX group. These results indicate that AEG-1 deletion can ameliorate inflammatory damage in ovariectomized rats with osteoporosis.

\section{AEG-1 deletion inbibits the TLR4/MyD88/NF- $\kappa B$ pathway to ameliorate inflammatory damage in ovariectomized rats with osteoporosis}

To investigate the associated pathway of the suppressive effects of AEG-1 deletion on osteoporosis, the expression of pathway proteins TLR4, MyD88 and NF- $\mathrm{BB}$ were measured by western blotting. As shown in Figure $5 A$, the expression of p-TLR4, p-MyD88 and p-NF- $\kappa$ B were all increased in the WT OVX group compared with the WT sham group. The overexpression and ratios of p-TLR4/

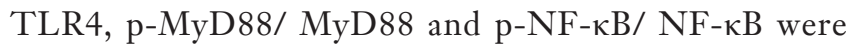
reversed in the AEG-1 KO OVX group.

To further verify the role of AEG-1 in the progression of osteoporosis, an LPS model of inflammatory osteoporosis was established. The expression of p-TLR4, p-MyD88 and $\mathrm{p}-\mathrm{NF}-\kappa \mathrm{B}$ were all enhanced in the WT OVX + LPS group compared with the WT OVX group. The overexpression and ratios of p-TLR4/ TLR4, p-MyD88/MyD88 and $\mathrm{p}-\mathrm{NF}-\kappa \mathrm{B} / \mathrm{NF}-\kappa \mathrm{B}$ were reversed in the AEG-1 KO OVX + LPS group (Figure 5B).

The protein expression levels of the bone specific transcription factors Osx, CoI1A1 and OC were measured by western blotting. As shown in Figure 5C, compared with the WT OVX group, the expression of Osx, CoI1A1 and OC were decreased in the WT OVX + LPS group. This aberrant expression was reversed in the AEG-1 KO OVX + LPS group.

The concentration of inflammatory factors IL-17 and IL-10 were measured with ELISA. Compared with the WT OVX group, the concentration of IL-17 was increased in the WT OVX + LPS group, whilst this increase was not present in the AEG-1 KO OVX + LPS group (Figure 5D). The concentration of IL-10 did not increase significantly 

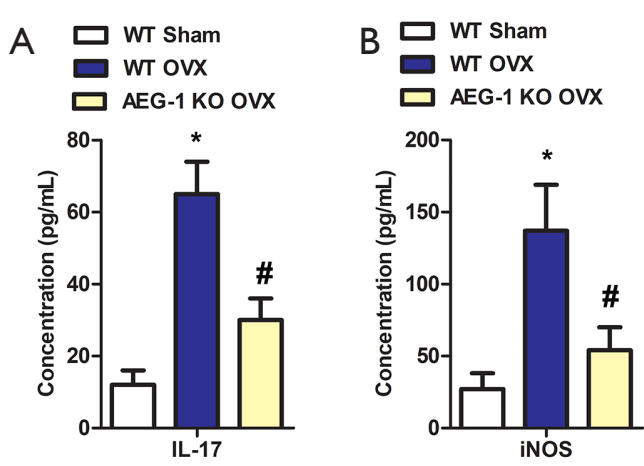

E
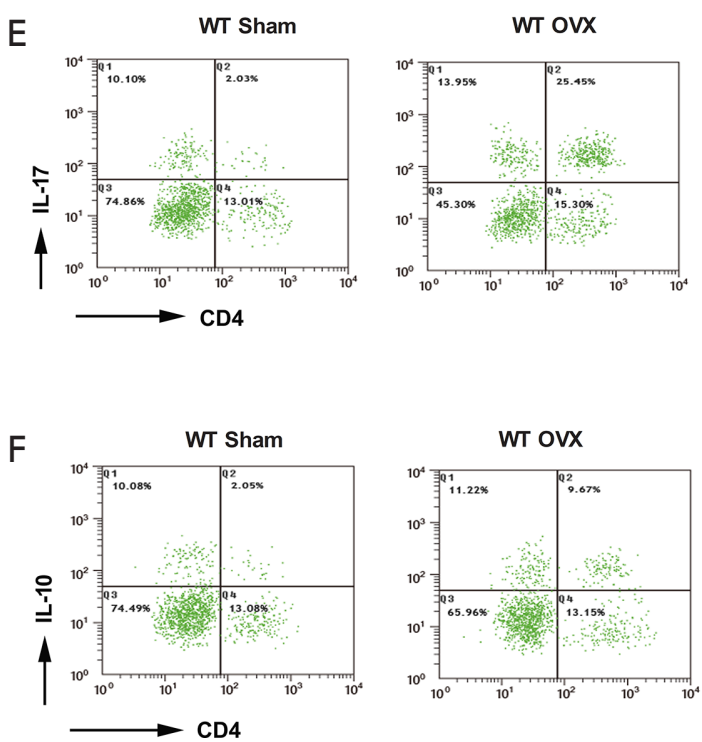
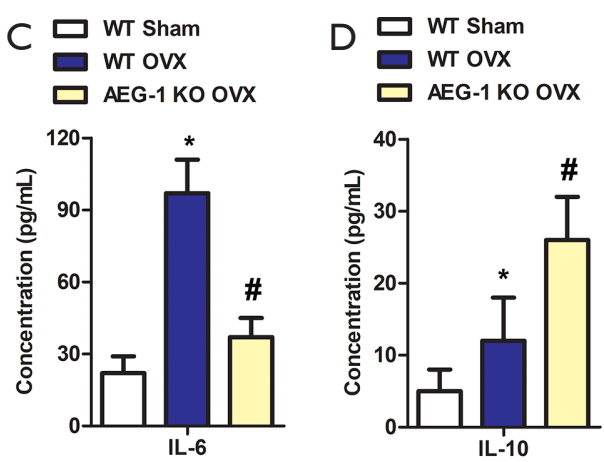

$$
\begin{aligned}
& \text { WT Sham } \\
& \text { WT ovx }
\end{aligned}
$$
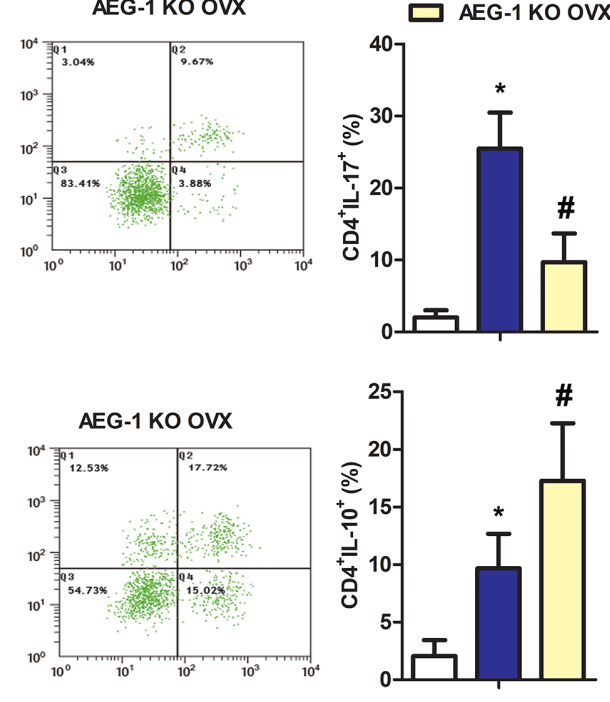

Figure 4 AEG-1 deletion ameliorates inflammatory damage in ovariectomized rats with osteoporosis. AEG-1 deletion decreases the concentrations of IL-17 (A), iNOS (B) and IL-6 (C) in peripheral blood. (D) AEG-1 deletion enhances the concentrations of IL-10 in peripheral blood. (E) AEG-1 deletion decreases the number of $\mathrm{CD} 4^{+} \mathrm{IL}-17^{+}$positive cells in peripheral blood. (F) AEG-1 deletion enhances the number of $\mathrm{CD}^{+} \mathrm{IL}-10^{+}$positive cells in peripheral blood. The results are presented as mean $\pm \mathrm{SD}$ and represent three average of individual experiments. *, $\mathrm{P}<0.05$ versus the WT Sham group $(\mathrm{n}=4)$; ${ }^{*}, \mathrm{P}<0.05$ versus the WT OVX group (n=4).

in the WT OVX + LPS group compared with the WT OVX group, however, increased significantly compared with the AEG-1 KO OVX + LPS group (Figure 5E). Collectively, AEG-1 deletion inhibits the TLR4/MyD88/ $\mathrm{NF}-\kappa \mathrm{B}$ pathway to ameliorate inflammatory damage in ovariectomized rats with osteoporosis.

\section{Discussion}

Osteoporosis includes several subtypes, and can be divided into both primary and secondary osteoporosis. Postmenopausal osteoporosis, in the category of primary osteoporosis, is the most common type of osteoporosis.
This contributes to the higher incidence of osteoporosis in women compared to men (23). Postmenopausal osteoporosis is caused by estrogen deficiency, leading to increased bone turnover (23). This ultimately leads to a loss of connectivity in trabecular bone and cortical pores of cortical bone $(24,25)$. Currently, treatment for postmenopausal osteoporosis mainly focuses on hormonal regulation, but is severely limited due to its side effect profile. In our study, WT and AEG-1 KO rats were used to establish an ovariectomy model. In our study, trabecular bone morphometry was aberrant, the expression of the bone specific transcription factors Osx, CoI1A1 and OC decreased, levels of IL-6, IL-17, iNOS increased and the TLR-4/MyD88/NF-kB 

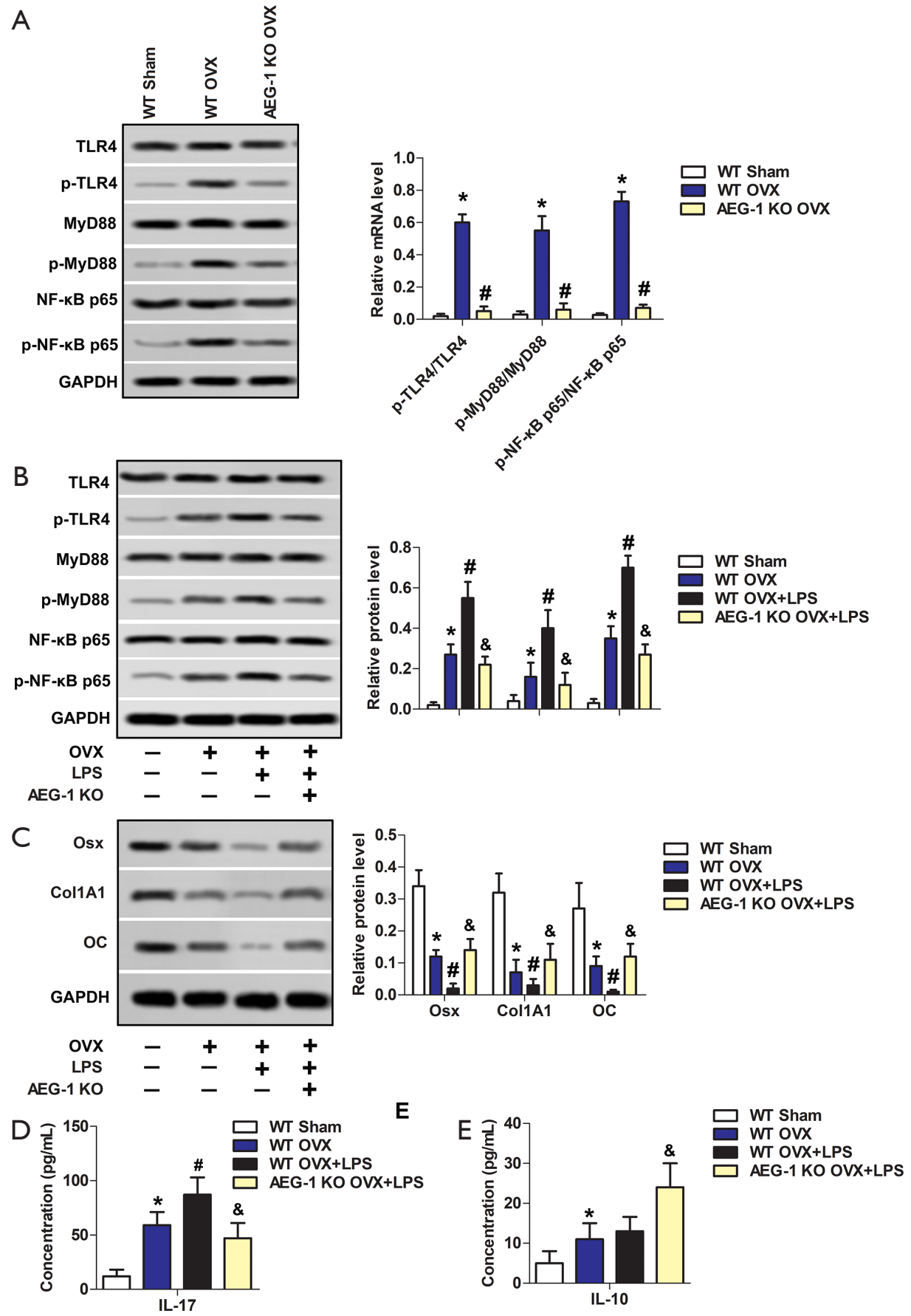

Figure 5 AEG-1 deletion inhibits the TLR4/MyD88/NF- $\kappa$ B pathway to ameliorate inflammatory damage in ovariectomized rats with osteoporosis. (A) AEG-1 deletion inhibits the ratios of p-TLR4/TLR4, p-MyD88/MyD88 and p-NF- $\kappa \mathrm{B}$ p65/NF- $\mathrm{BB}$ p65 in ovariectomized

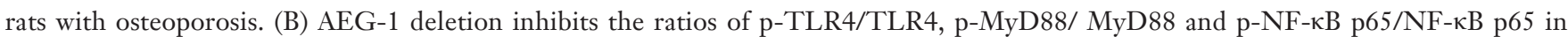
ovariectomized and LPS-induced rats with osteoporosis. (C) AEG-1 deletion decreases the relative protein levels of Osx, Col1a1 and OC in ovariectomized and LPS-induced rats with osteoporosis. (D) AEG-1 deletion decreases the concentration of IL-17 in ovariectomized and LPS-induced rats with osteoporosis. (E) AEG-1 deletion enhances the concentration of IL-10 in ovariectomized and LPS-induced rats with osteoporosis. The results are presented as mean $\pm \mathrm{SD}$ and represent the average of three individual experiments. *, $\mathrm{P}<0.05$ versus the $\mathrm{WT}$ sham group ( $n=4) ;{ }^{\#}, \mathrm{P}<0.05$ versus the WT OVX group $(\mathrm{n}=4) ;{ }^{*}, \mathrm{P}<0.05$ versus the WT OVX + LPS group (n=4). 
signaling pathway was activated. Of note, AEG-1 deletion alleviated these aberrant phenomena and reversed LPS induced damage.

Research has revealed that AEG-1 plays a critical role in aggressive tumors, including metastasis, invasion, angiogenesis and evasion of apoptosis (26). Zhang et al. reported that AEG-1 was overexpressed in osteosarcoma cells and miR-342-3p mitigated the proliferation, migration and invasion of osteosarcoma cells by targeting AEG-1 (27). However, the expression of AEG-1 in osteoporosis had not yet been reported. In the present study, AEG-1 was overexpressed in an ovariectomized rat model, and AEG-1 participated in the pathological process of postmenopausal osteoporosis. Importantly, we found that AEG-1 deletion improved trabecular bone morphology and increased the bone mass in an osteoporosis model. These results indicate that targeting AEG-1 may be a novel therapeutic approach for osteoporosis treatment.

Cartilage tissue is simple in composition, complex in structure and difficult to repair after injury. Adult articular cartilage has limited ability to fully self-repair due to a lack of revascularization, and therefore, impaired access to regenerative cells (28). In recent years, scientists have continued to study auxiliary methods for cartilage repair. Zhang et al. revealed that mesenchymal stem cell exosomes mediate cartilage repair by enhancing proliferation, attenuating apoptosis and modulating immune reactivity (29). Zhou et al. reported that Berberine-mediated release of surfactant protein D (SP-D) promoted cartilage repair by modulating immune responses via the inhibition of TLR4/NF-кB signaling (30). Bone remodeling is closely related to osteoblast differentiation and osteoclast resorption, which are important processes in the treatment of osteoporosis. In our study, shrunken nucleus pulposus, cluster formation and cleft formation with fissures was observed in the WT OVX group. The percentage of trabecular bone and cartilage area were significantly decreased in the WT OVX group compared to the WT sham group. Furthermore, the expression of the bone specific transcription factors Osx, CoI1A1 and OC increased in the WT OVX group compared to the WT sham group. We also found that the levels of inflammatory factors including IL-6, iNOS, IL-17 and the anti-inflammatory cytokine IL-10 significantly increased in WT OVX rats compared with the WT sham group. Notably, AEG-1 deletion reversed these adverse reactions in the AEG-1 KO OVX group. These results suggest that AEG-1 deletion mitigates the adverse reactions induced by osteoporosis.
LPS, found in Gram-negative bacteria, is a potent initiator of inflammatory responses. LPS triggers TLR4mediated signaling pathways including NF- $\mathrm{kB}, \mathrm{PI} 3 \mathrm{~K} / \mathrm{Akt}$ and a spectrum of mitogen-activated protein kinases $(31,32)$. These signaling pathways ultimately lead to a cellular response, followed by production of proinflammatory cytokines and other mediators. Sun et al. reported that LPS induction can cause inflammatory bone destruction, and pregnenolone inhibited this phenomenon (20). In our study, levels of IL-17 in WT OVX rats increased after exposure to LPS. The expression level of the antiinflammatory cytokine IL-10 did not change significantly compared to the WT sham group. Furthermore, we found that LPS induced the activation of TLR4, MyD88 and NF$\kappa \mathrm{B}$ signaling pathways. Researchers revealed that AEG-1 participates in the activation of NF- $\mathrm{BB}(33,34)$, and LPSinduced cytokine production is mainly dependent on NF$\kappa \mathrm{B}$ activation $(35,36)$. Consistent with our study, Khuda et al. demonstrated that AEG-1 could be classified as a LPS-responsive gene and is involved in the LPS-induced inflammatory response (37). In addition, LPS can induce AEG-1 as a TLR4 ligand, and can regulate TLR4 signaling. Importantly, we found that AEG-1 deletion reversed the adverse reactions induced by LPS, providing further evidence of the role of AEG-1 in osteoporosis.

In conclusion, the results of present study suggest that targeting AEG-1 may be a potential therapeutic strategy for osteoporosis. We found that AEG-1 deletion can mitigate bone histopathological damage, reduce bone loss and promote the expression of osteogenic marker proteins. The underlying mechanism involves the inhibition of the TLR4/MyD88/NF- $\mathrm{B}$ pathway. It should be noted that the present study was implemented in animal models with a limited sample size. Therefore, further in depth studies are needed to verify the preventive effect of AEG-1 deletion on osteoporosis.

\section{Acknowledgments}

Funding: None.

\section{Footnote}

Reporting Checklist: The authors have completed the ARRIVE reporting checklist. Available at http://dx.doi. org/10.21037/atm-20-5842

Data Sharing Statement: Available at http://dx.doi. 
org/10.21037/atm-20-5842

Conflicts of Interest: Both authors have completed the ICMJE uniform disclosure form (available at http://dx.doi. org/10.21037/atm-20-5842). The authors have no conflicts of interest to declare.

Ethical Statement: The authors are accountable for all aspects of the work in ensuring that questions related to the accuracy or integrity of any part of the work are appropriately investigated and resolved. All animal experiment protocols were approved by Roslin Institute's Animal Users Committee (A650). All animal experiments were performed in accordance with the International Guiding Principles for Biomedical Research Involving Animals issued by the Council for the International Organizations of Medical Sciences.

Open Access Statement: This is an Open Access article distributed in accordance with the Creative Commons Attribution-NonCommercial-NoDerivs 4.0 International License (CC BY-NC-ND 4.0), which permits the noncommercial replication and distribution of the article with the strict proviso that no changes or edits are made and the original work is properly cited (including links to both the formal publication through the relevant DOI and the license). See: https://creativecommons.org/licenses/by-nc-nd/4.0/.

\section{References}

1. Glaser DL, Kaplan FS. Osteoporosis. Definition and clinical presentation. Spine 1997;22:12S-6S.

2. Chen X, Zhang S, Chen X, et al. Emodin promotes the osteogenesis of MC3T3-E1 cells via BMP-9/Smad pathway and exerts a preventive effect in ovariectomized rats. Acta Biochim Biophys Sin (Shanghai) 2017;49:867-78.

3. Wang XR, Kwok TCY, Griffith JF, et al. Prevalence of cervical spine degenerative changes in elderly population and its weak association with aging, neck pain, and osteoporosis. Ann Transl Med 2019;7:486.

4. Lundberg AM, Ketelhuth DF, Johansson ME, et al. Toll-like receptor 3 and 4 signalling through the TRIF and TRAM adaptors in haematopoietic cells promotes atherosclerosis. Cardiovasc Res 2013;99:364-73.

5. Chimenti C, Verardo R, Scopelliti F, et al. Myocardial expression of Toll-like receptor 4 predicts the response to immunosuppressive therapy in patients with virus-negative chronic inflammatory cardiomyopathy. Eur J Heart Fail
2017;19:915-25.

6. Lu M, Tang F, Zhang J, et al. Astragaloside IV attenuates injury caused by myocardial ischemia/reperfusion in rats via regulation of toll-like receptor $4 /$ nuclear factor- $\kappa \mathrm{B}$ signaling pathway. Phytother Res 2015;29:599-606.

7. Soraya H, Clanachan AS, Rameshrad M, et al. Chronic treatment with metformin suppresses toll-like receptor 4 signaling and attenuates left ventricular dysfunction following myocardial infarction. Eur J Pharmacol 2014;737:77-84.

8. Zhang J, Zhang J, Yu P, et al. Remote Ischaemic Preconditioning and Sevoflurane Postconditioning Synergistically Protect Rats from Myocardial Injury Induced by Ischemia and Reperfusion Partly via Inhibition TLR4/MyD88/NF-кB Signaling Pathway. Cell Physiol Biochem 2017;41:22-32.

9. Ma SR, Xie XW. NLRC5 deficiency promotes myocardial damage induced by high fat diet in mice through activating TLR4/NF-kB. Biomed Pharmacother 2017;91:755-66.

10. Wang Q, Lin P, Li P, et al. Ghrelin protects the heart against ischemia/reperfusion injury via inhibition of TLR4/NLRP3 inflammasome pathway. Life Sci 2017;186:50-8.

11. Vijayan V, Khandelwal M, Manglani K, et al. Methionine down-regulates TLR4/MyD88/NF- $\kappa B$ signalling in osteoclast precursors to reduce bone loss during osteoporosis. Br J Pharmacol 2014;171:107-21.

12. Su ZZ, Kang DC, Chen Y, et al. Identification and cloning of human astrocyte genes displaying elevated expression after infection with HIV-1 or exposure to HIV-1 envelope glycoprotein by rapid subtraction hybridization, $\mathrm{RaSH}$. Oncogene 2002;21:3592-602.

13. Kang DC, Su ZZ, Sarkar D, et al. Cloning and characterization of HIV-1-inducible astrocyte elevated gene-1, AEG-1. Gene 2005;353:8-15.

14. Yoo BK, Emdad L, Lee SG, et al. Astrocyte elevated gene1 (AEG-1): A multifunctional regulator of normal and abnormal physiology. Pharmacol Ther 2011;130:1-8.

15. Ying Z, Li J, Li M. Astrocyte elevated gene 1: biological functions and molecular mechanism in cancer and beyond. Cell Biosci 2011;1:36.

16. Luo Y, Zhang X, Tan Z, et al. Astrocyte Elevated Gene-1 as a Novel Clinicopathological and Prognostic Biomarker for Gastrointestinal Cancers: A Meta-Analysis with 2999 Patients. PLoS One 2015;10:e0145659.

17. Sarkar D, Emdad L, Lee SG, et al. Astrocyte elevated gene-1: far more than just a gene regulated in astrocytes. Cancer Res 2009;69:8529-35. 
18. Song L, Li W, Zhang H, et al. Over-expression of AEG1 significantly associates with tumour aggressiveness and poor prognosis in human non-small cell lung cancer. J Pathol 2009;219:317-26.

19. Wang F, Pang JD, Huang LL, et al. Nanoscale polysaccharide derivative as an AEG-1 siRNA carrier for effective osteosarcoma therapy. Int J Nanomedicine 2018;13:857-75.

20. Sun X, Zhang C, Guo H, et al. Pregnenolone Inhibits Osteoclast Differentiation and Protects Against Lipopolysaccharide-Induced Inflammatory Bone Destruction and Ovariectomy-Induced Bone Loss. Front Pharmacol 2020;11:360.

21. Cui Q, Xing J, Yu M, et al. Mmu-miR-185 depletion promotes osteogenic differentiation and suppresses bone loss in osteoporosis through the Bgn-mediated BMP/Smad pathway. Cell Death Dis 2019;10:172.

22. Quintarelli G, Dellovo MC. The chemical and histochemical properties of alcian blue. IV. Further studies on the methods for the identification of acid glycosaminoglycans. Histochemie 1965;5:196-209.

23. Sharma N, Natung T, Barooah R, et al. Effect of Multiparity and Prolonged Lactation on Bone Mineral Density. J Menopausal Med 2016;22:161-6.

24. Diab DL, Watts NB. Postmenopausal osteoporosis. Curr Opin Endocrinol Diabetes Obes 2013;20:501-9.

25. Black DM, Rosen CJ. Clinical Practice. Postmenopausal Osteoporosis. N Engl J Med 2016;374:254-62.

26. Hu B, Thirtamara-Rajamani KK, Sim H, et al. Fibulin-3 is uniquely upregulated in malignant gliomas and promotes tumor cell motility and invasion. Mol Cancer Res 2009;7:1756-70.

27. Zhang S, Liu L, Lv Z, et al. MicroRNA-342-3p Inhibits the Proliferation, Migration, and Invasion of Osteosarcoma Cells by Targeting Astrocyte-Elevated Gene-1 (AEG-1). Oncol Res 2017;25:1505-15.

Cite this article as: Zhang Y, Zhao Q. AEG-1 deletion promotes cartilage repair and modulates bone remodelingrelated cytokines via TLR4/MyD88/NF- $\kappa$ B inhibition in ovariectomized rats with osteoporosis. Ann Transl Med 2020;8(20):1298. doi: 10.21037/atm-20-5842
28. O'Driscoll SW. The healing and regeneration of articular cartilage. J Bone Joint Surg Am 1998;80:1795-812.

29. Zhang S, Chuah SJ, Lai RC, et al. MSC exosomes mediate cartilage repair by enhancing proliferation, attenuating apoptosis and modulating immune reactivity. Biomaterials 2018;156:16-27.

30. Zhou Y, Ming J, Deng M, et al. Berberine-mediated upregulation of surfactant protein $\mathrm{D}$ facilitates cartilage repair by modulating immune responses via the inhibition of TLR4/NF-KB signaling. Pharmacol Res 2020;155:104690.

31. Takeda K, Kaisho T, Akira S. Toll-like receptors. Annual review of immunology 2003;21:335-76.

32. Medvedev AE, Sabroe I, Hasday JD, et al. Tolerance to microbial TLR ligands: molecular mechanisms and relevance to disease. J Endotoxin Res 2006;12:133-50.

33. Emdad L, Sarkar D, Su ZZ, et al. Activation of the nuclear factor kappaB pathway by astrocyte elevated gene-1: implications for tumor progression and metastasis. Cancer Res 2006;66:1509-16.

34. Sarkar D, Park ES, Emdad L, et al. Molecular basis of nuclear factor-kappaB activation by astrocyte elevated gene-1. Cancer Res 2008;68:1478-84.

35. Manthey CL, Perera PY, Henricson BE, et al. Endotoxininduced early gene expression in $\mathrm{C} 3 \mathrm{H} / \mathrm{HeJ}$ (Lpsd) macrophages. J Immunol1994;153:2653-63.

36. Yamamoto M, Sato S, Hemmi H, et al. Essential role for TIRAP in activation of the signalling cascade shared by TLR2 and TLR4. Nature 2002;420:324-9.

37. Khuda II, Koide N, Noman AS, et al. Astrocyte elevated gene-1 (AEG-1) is induced by lipopolysaccharide as toll-like receptor 4 (TLR4) ligand and regulates TLR4 signalling. Immunology 2009;128:e700-6.

(English Language Editor: C. Betlazar-Maseh) 